effect on international collaboration (see Nature 457, 511; 2009, and 447, 890-891; 2007). In the short term, academic freedom and the isolation of Iran's scientists are likely to worsen under a regime desperate to cling onto power by crushing reformist elements, and targeting thousands of students, academics and other intellectuals for arrest. Seventy academics were arrested on 24 June after meeting Mousavi to give their analysis of current Iranian society, and other academics are reported to have been detained since, although reliable information is scarce. Academics are now in the front line, says one Iranian researcher who returned from Iran just days ago.

In the face of this bleak news, however, Iranian academics are surprisingly optimistic. They tend to buy into the argument that, despite the current crackdown, greater democracy is inevitable in Iran, which will provide an open society that is more conducive to science and critical thinking. They point out that Iran and Turkey are the two Muslim countries with the strongest democratic and secular traditions - and that academics have played a major part in helping the society resist religious obscurantism. Iran is not the only country in the region where human rights and democracy are violated; and the West has hypocritically been relatively silent on similar abuses by several of its allies in the Middle East. But in Iran at least, the country's long traditions of democracy, education and free thinking - suppressed for decades by the regime, and in particular the current hard-line leadership - are now out in the open.

\section{Time for early action}

\section{Carbon dioxide is not the only warming agent worth tackling now in the bid to cool the planet.}

T

he US House of Representatives must be commended for passing a comprehensive climate bill last week that would finally set the United States on a path to lower its greenhouse-gas emissions. The pending legislation is far from perfect and will face a tough test in the Senate. But it is a necessary first step for the country that has so far added the most carbon dioxide to the atmosphere.

However, it will take several decades, if not longer, for the United States and other nations to significantly reduce carbon dioxide emissions. This fact has spurred many scientists to intensify research into techniques that might provide a more immediate way to turn down the planetary thermostat. Some solutions seek to fine-tune Earth's climate through large-scale geoengineering projects, such as pumping sulphates into the atmosphere to reflect more sunlight back into space. But another approach - an 'early action' climate agenda increasingly being pushed by environmentalists and some scientists - might prove safer and much easier to sell to governments and populations around the world.

A good place to start is with black carbon, the sooty, dark component of smoke that emanates from diesel engines, inefficient cooking stoves, forest fires and the like. Black carbon is a danger to human health, having been implicated in a variety of cardiovascular and respiratory diseases, and may also be a major player when it comes to global warming, particularly in regions that spend at least part of the year covered with ice and snow (see page 29). But unlike carbon dioxide, which stays in the atmosphere for centuries, black-carbon particles remain in the air for just a matter of weeks. So, in principle, efforts to eliminate emissions could quickly reduce the warming power of this pollutant.

Unfortunately, it is not that simple. First, not all black carbon is anthropogenic in origin. Second, black carbon is accompanied to varying degrees by its lighter-coloured cousin, organic carbon, which cools the planet along with most other reflective aerosols. Third, despite more than a decade of research, the chain of reactions by which black carbon warms the atmosphere and melts snow remains surprisingly hazy. All of these factors make black carbon's effect on climate difficult to quantify.
However, none of the caveats is a reason for nations not to try their utmost to control it. Even if the climate benefits turn out to be less than hoped, cleaner air would save hundreds of thousands of lives a year. Governments should already be working to clean up diesel emissions and to improve cooking stoves in southeast Asia, where the health problems are most acute. But it may be that the threat of global warming is more effective than health advocacy.

Accompanying black carbon on the early-action agenda are methane and hydrofluorocarbons (HFCs). The latter are used predominantly as refrigerants, and can be more than a thousand times more powerful than $\mathrm{CO}_{2}$ as greenhouse gases (see Nature 459, 1040-1041; 2009).

The international 'Methane to Markets' programme provides money and expertise to help countries to capture methane from sources such as landfills, farms and coal mines. Once up and running, these projects produce energy at a profit by making use of a clean-burning compound that would otherwise have wreaked havoc in the atmosphere for years. The programme has been a resounding success, and other nations should find the money and will to replicate it.

The case for HFCs is a bit more complicated, but no less strong. HFCs were developed to replace ozone-depleting chlorofluorocarbons that were phased out in response to the 1989 Montreal Protocol. Because they don't affect atmospheric ozone levels, they were pulled under the United Nations' Kyoto climate treaty. But there are now calls to take the chemicals out of the climate treaty and put them into the Montreal Protocol. This is the right thing to do. Montreal regulators have already proved their ability to implement worldwide curbs on emissions, and there is little doubt that they could handle this problem faster and more cost-effectively than could a cumbersome treaty aimed at targeting $\mathrm{CO}_{2}$.

Notably, each of these solutions could be pursued immediately, precisely because they make sense on multiple levels; global warming might be a driving factor, but it is not the only one. There is no need to wait for international negotiators to strike a deal on a climate treaty that would lay the groundwork for a global carbon market. And they do not pose the ethical or legal challenges that geoengineering schemes so often face.

Some fear that even talking about such subjects could distract from the main problem, which is $\mathrm{CO}_{2}$. The opposite is true. Providing workable solutions in other areas will build momentum and simultaneously ease the burden that remains. What is there to lose? 